widely used but its safety in children has not been established. Reasons for drug treatment failure include inadequate initial dosage and maintenance, undiscovered cause or complication, and misdiagnosis.(Shorvon S. Tonic clonic status epilepticus. I Neurol Neurosurg Psychiatry Feb 1993; 56: 125-134). (Respond: Dr Shorvon, Institute of Neurology, Queen Square, London WC1N 3BG, UK).

COMMENT. The annual incidence of new cases of tonic clonic status in the USA is estimated at $45,000-70,000$. It occurs in $10-25 \%$ of children with epilepsy. Mortality is $5-10 \%$, and morbidity increases with the duration of the status episode. Successful treatment of this medical emergency depends on the balance of a rapid control of seizures and the avoidance of complications of therapy. (Shorvon SD. Status epilepticus: its clinical features and treatment in children and adults. Cambridge, Cambridge University Press, 1993).

Phenytoin monitoring in status epilepticus in infants and children is reported from the Service de Neuropediatrie, Hopital SaintVincent de Paul, Paris, and Hopital du Bocage, Dijon, France. (Richard

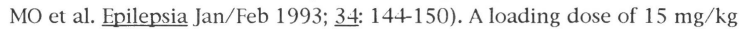
was followed by three injections in the first 24 hours. Monitoring with nine plasma samples during the first day allowed for dosage adjustment with increased efficacy and reduced toxicity. Older children responded better than younger children who had lower plasma levels.

\title{
CARBAMAZEPINE-INDUCED ACUTE PANCREATITIS
}

A 5-year-old mentally retarded child who developed pancreatitis during accidental acute carbamazepine (CBZ) intoxication is reported from the Department of Pediatrics, Ohio State University, Columbus, Ohio. He presented with vomiting and lethargy, but no abdominal pain. Serum amylase and lipase levels were increased for several days, and they returned to normal on recovery. (Tsao CY, Wright FS. Acute chemical pancreatitis associated with carbamazepine intoxication. Epilepsia Jan/Feb 1993; $\underline{34}$ : 174-176). (Reprints: Dr CY Tsao, Department of Pediatrics, Ohio State University, Children's Hospital, 700 Children's Dr, Columbus, $\mathrm{OH}$ 43205).

COMMENT. Among antiepileptic drugs, valproate is the most likely to be associated with pancreatitis. In a study at the Bowman Gray School of Medicine, Winston Salem, North Carolina, and the University of Virginia, Charlottesville, $14.5 \%$ of 366 physicians treating epilepsy reported a case of valproateassociated pancreatitis. Thirty nine cases were available for review from the physicians surveyed, the authors' patient population, and from the medical literature. Pancreatitis was more common in young persons (mean age 16 years) and during the early months of treatment. Three deaths were reported. Asymptomatic elevation of serum amylase was observed by $40(11 \%)$ of the 
physicians using valproate. (Asconape JJ, Penry JK, Dreifuss FE et al. Valproate-associated pancreatitis. Epilepsia Jan/Feb 1993; 34: 177-183).

\section{NEONATAL NEUROLOGY}

\section{PROGNOSIS OF INTRAVENTRICULAR HEMORRHAGE}

The relationship of periventricular-intraventricular hemorrhage in infants of $<32$ weeks gestation to the occurrence of disability at 5 years of age was studied in a National Collaborative Survey in the Netherlands. Of 484 infants enrolled, all 304 survivors were examined at age 5 years; $26 \%$ of infants with severe hemorrhage and $67 \%$ with mild grades I/II survived the neonatal period. Of $85(28 \%)$ survivors with a disability, $27(9 \%)$ had a minor handicap and $23(8 \%)$ a major handicap. The handicap was recorded at the age of 5 but was not present at 2 years in 17 children, while 35 were handicapped at 2 years but not at 5 years. Children with grades I and II as well as III and IV had more disabilities and handicaps than children without hemorrhage. (van de Bor $\mathrm{M}$ et al. Outcome of periventricular-intraventricular haemorrhage at five years of age. Dev Med Child Neurol Jan 1993; 35 : 33-41). (Respond: Dr van de Bor, Division of Neonatology, Department of Paediatrics, University Hospital Leiden, PO Box 9600, 2300 RC Leiden, The Netherlands).

COMMENT. The small number of survivors with grades III and IV hemorrhage was attributed to the widely used policy in the Netherlands of withdrawing life-support from infants with severe cerebral damage. Of 65 in this category, 47 died in the neonatal period. At the 5 year evaluation, only $25 \%$ of the patients had had severe hemorrhage. Children with only mild hemorrhage had a significantly increased risk of disability at the age of 5 years. Patients with evidence of periventricular leukomalacia were excluded from this study.

\section{LOCAL ANESTHESIA IN NEONATAL LUMBAR PUNCTURE}

The success rate of lumbar punctures was compared in neonates allocated prospectively to 0.2 to $0.5 \mathrm{~mL}$ of $1 \%$ lidocaine anesthesia $(\mathrm{n}=48)$ or a control group $(\mathrm{n}=52)$ in a study at the Albany Medical College of Union University, Albany, New York. A struggling motion score increased during the lidocaine injection but decreased at the spinal needle insertion. Despite the decreased motion following lidocaine, the number of attempts per lumbar puncture, failure rate, and traumatic punctures were not different from the controls. The success rate was not dependent on the level of physician training. (Pinheiro JMB et al. Role of local anesthesia during lumbar puncture in neonates. Pediatrics Feb 1993; 91: 379-382). (Reprints: Dr Pinheiro, Dept of Pediatrics, Albany Medical Center A-101, Albany, NY 12208)

COMMENT. The authors advocate lidocaine anesthesia in neonatal LPs. 